\title{
DIRECTION OF ARRIVAL ASSESSMENT IN AIRBORNE ICE-SOUNDING SYNTHETIC APERTURE RADAR
}

\author{
Alvaro Arenas-Pingarron ${ }^{1}$, Paul V. Brennan ${ }^{1}$, Hugh Corr $^{2}$ \\ ${ }^{1}$ Department of Electronic \& Electrical Engineering, University College London, London WC1E 7 JE, UK \\ ${ }^{2}$ British Antarctic Survey, High Cross, Madingley Road, Cambridge CB3 0ET, UK
}

\begin{abstract}
We propose an algorithm to evaluate the direction of arrival (DoA) estimation in the cross-track dimension of an airborne pulsed ice-sounding synthetic aperture radar (SAR), formed by a non-linear array. The conventional methods process either simulated data, meaning a lack of real scenario, or collected over regions previously mapped, where a limited number of DoAs is found. In our technique, we combine the echoes of different transmitted pulses from a real data take above the sea surface, planned to vary the roll angle of the aircraft. Due to the mirror-like behavior of the sea, when echoes at different roll angles are summed, we obtain raw data or images with several DoAs, relative to the main axes of the aircraft. This method is used to assess the estimation algorithms and choose the optimal for Antarctic bedrock 3Dimaging, identifying the DoAs to shape the true bed topography.
\end{abstract}

Index Terms - Direction-of-arrival estimation, icesounding, MIMO radar, MUSIC, SAR.

\section{INTRODUCTION}

To resolve the DoA within the beam of the radiation pattern, a 3D ice-sounding synthetic aperture radar uses an array of antennas along the cross-track direction. In the airborne case, the array elements are typically attached under the wings and main aircraft body. Due to the small number of elements in the array, linear estimators like beam-steering [1] and MVDR (Minimum Variance Distortionless Response) [1-2] are limited in resolution, and high-resolution approaches based on autoanalysis like MUSIC (MUltiple SIgnal Classification) [2-4] and maximum likelihood [5] are preferred. In case the antennas follow a non-uniform distribution, the estimator performance is strongly affected, and the algorithms should be assessed to check the output under several conditions, and to develop new approaches to improve the results.

The main strategy to evaluate the DoA estimators is to simulate the impinging signals. Regardless the flexibility for including several scatterers in arbitrary range cells, the main problem is that it does not consider the relative amplitude and phase mismatch between channels due to calibration errors, and the results may be inaccurate. To work under the conditions of a real scenario, a possibility is to deploy signal reflectors on the surface field, difficult for the case of polar ice-sounding. As an alternative, in well-known regions pointand extended-like opportunity targets can be found. They arise from geographical features, which act as effective signal reflectors, like for example human-made structures with double-bounce reflections or surface slopes perpendicular to the incidence angle. These cases were found in calibration flights above Rothera Research Station (a British facility in Adelaide Island, in the Antarctic Peninsula), but due to the concave elevation profile of the region and the number of infrastructures, it was difficult to resolve the many DoAs.

Another source for opportunity scatterers are the water masses because its mirror-like electromagnetic wave reflection. In the case of sea water, when the signal wavelength is long regarding the sea waves, the sea is regarded as a flat surface, and the signals follow a specular path from the transmitter (TX) to the receiver (RX). This case was exploited in [6] with a multiple-input multiple-output (MIMO) airborne ice-sounding SAR at $150 \mathrm{MHz}$, to obtain the effective TX antenna pattern and check the condition of all TX/RX elements.

\section{MODEL DESCRIPTION}

The data were taken with the British Antarctic Survey (BAS) PASIN2 (Polarimetric Airborne Scientific Instrument, mark 2), a pulsed MIMO SAR at $150 \mathrm{MHz}$ (wavelength $\lambda=2 \mathrm{~m}$ ) with folded dipoles, having 2 effective TXs (one per wing) and 12 independent RXs. Both TXs are formed by an array of 4 elements below each wing, also configured as RXs (after a transmission window interval). The remaining 4 only-RX elements are in a radome beneath the fuselage (belly), off the wing antennas alignment. Port elements are labelled as P1, P2, P3, P4; belly as B5, B6, B7, B8; and starboard as S9, SA, $\mathrm{SB}, \mathrm{SC}$. In this paper, only the port TX was used. The aircraft rotation angles are roll (around the direction of movement ' $x$ ', positive angle according to the right-hand rule), pitch (around port direction ' $y$ ', positive according to the left-hand rule) and yaw (around direction opposite to gravity force ' $z$ ', positive according to the left-hand rule). The antenna distribution ( $x_{n}$, $y_{n}, z_{n}$ ) is shown in Fig. 1, whose locations in meters (rounded to $\mathrm{cm}$ ) are in Table I, together with the distance normalized to the wavelength $\left(d_{n} / \lambda\right)$ of the imaginary segment joining two 


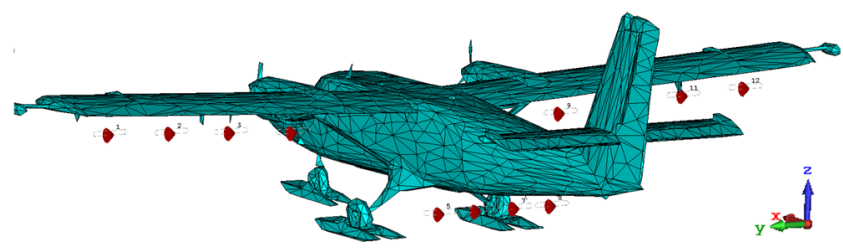

Fig. 1. PASIN2 scanned model, with antenna elements as cones.

consecutive elements from port to starboard, and its angle $\beta_{n}(0)$ regarding ' $x y$ ' plane, zero when perpendicular to nadir and negative clockwise. Regarding the DoA, its angle $\alpha$ is positive when the signal comes from port and zero from nadir.

Table I. Cartesian coordinates $(x, y, z)$ of antenna locations $(\mathrm{m})$, electrical distance $d_{n} / \lambda$ and angle $\beta_{n}(0)$ regarding ' $x y$ ' plane of the segments joining consecutive antenna elements.

\begin{tabular}{|c|c|c|c|c|c|c|c|c|c|c|c|c|}
\cline { 2 - 10 } \multicolumn{1}{c|}{} & P1 & P2 & P3 & P4 & B5 & B6 & B7 & B8 & S9 & SA & SB & SC \\
\hline$x_{n}$ & 2.33 & 2.31 & 2.33 & 2.32 & -0.59 & -0.59 & -0.59 & -0.59 & 2.32 & 2.32 & 2.32 & 2.33 \\
$y_{n}$ & 8.36 & 6.75 & 5.15 & 3.53 & 1.46 & 0.49 & -0.52 & -1.48 & -3.52 & -5.14 & -6.75 & -8.38 \\
$z_{n}$ & 1.18 & 1.11 & 1.02 & 0.92 & -0.59 & -0.59 & -0.59 & -0.59 & 0.92 & 1.02 & 1.11 & 1.18 \\
\hline$d_{n} / \lambda$ & 0.81 & 0.80 & 0.81 & 1.94 & 0.49 & 0.51 & 0.48 & 1.93 & 0.81 & 0.81 & 0.82 \\
$\beta_{n}(0)$ & $-2.5^{\circ}$ & $-3.2^{\circ}$ & $-3.5^{\circ}$ & $-23^{\circ}$ & $0^{\circ}$ & $0^{\circ}$ & $0^{\circ}$ & $23^{\circ}$ & $3.5^{\circ}$ & $3.2^{\circ}$ & $2.5^{\circ}$ \\
\hline
\end{tabular}

Since the length of the receiving array is several orders of magnitude smaller than the height above the surface, the highest amplitude peak at each RX comes from a direction perpendicular to the sea surface and the same resolution cell. The overall effect is an instantaneous (for each transmitted pulse) point-like target with a DoA relative to the main axes of the aircraft and dependent mainly on the roll angle. According to the angle conventions, when the aircraft roll angle is positive (starboard wing lower than port), after rangeprocessing the received echoes and assuming zero roll angle, the resulting DoA is negative (starboard).

The calibration/validation (cal/val) flights are planned above the sea in the nearby of Rothera Station, if possible before and after the scientific imaging flights with common setups. The main drawback of these techniques based on the region overflown is that they cannot be applied in periodical basis within each data take, and they require a fixed setup. In the case of PASIN2, the rolling calibration flight was first included in the 16/17 campaign, but it will be a standard procedure to compare the performances in the next missions.

\section{SYNTHETIC DATA GENERATION}

The point-like targets were obtained in a data take above the Antarctic Sea, during a calibration flight in which the roll angle was planned to vary $\pm 25^{\circ}$ around the vertical, with a circumstantial pitch about $+5^{\circ}$.

A validation of the TX antenna pattern and RX elements must be performed, to know which of them to use for the DoA estimation. The procedure is equivalent to the followed in [6], normalizing to the maximum the peak response (produced by the different transmission modes) at each receiver, against the roll angle measured by an inertial measurement unit (IMU). The resulting normalized pairs of amplitude/roll angle for each RX and TX should resemble the cross-track TX-array pattern, and the relative amplitude among the RX's should be consistent except for the antenna nulls.

Next step is the mismatch corrections of TX and RX channels, produced by multipath effects or different electrical lengths, whose resulting relative amplitude and phase values for each channel can be estimated from the same cal/val flight. This calibration procedure is simplified assuming constant correcting factors, regardless the aircraft orientation and DoA. For evaluating the calibration, the estimated DoA from the peak responses at each RX is compared before and after the corrections. Due to the limited number of channels, the chosen estimator is MUSIC, with unbiased single DoA (regarding the opposite value of the roll angle, according to the angle convention in this paper) and lower sidelobes after the constant complex (amplitude and phase) correction, as shown in Fig. 2, where the results before and after calibration are in the top and bottom images, respectively, with the roll angle measured from the IMU (solid line), against the transmitted pulse number. The results, in $\mathrm{dB}$, are normalized to the maximum of each pulse, and hence in the null of the TX pattern (around pulses 1500, 2900, 4600 and 6900) the results appear as black strips.

Considering the SAR data from sea scattering as linear in range and azimuth because its narrow doppler bandwidth (due to the mirror-like reflection), to generate a certain number of DoAs the same number of regions of raw data or SAR images can be overlapped. Since the height over surface varies, the simple addition of raw data or images does not result in point-like target with constant range. After antenna pattern and height compensations, sections of processed image with different roll angle are summed to approach multiple DoAs in the same range and with equal amplitude, allowing to assess the estimators under real conditions.

\section{DIRECTION OF ARRIVAL ESTIMATION}

MUSIC algorithm [3] offers high-resolution estimations of the DoA, but its non-linear properties make it a qualitative procedure, rather than quantitative. It spans the received data into signal $(S)$ and noise $(N)$ orthogonal subspaces, obtained by the singular value decomposition (SVD) of the correlation matrix estimated from the received data, classifying the algorithm into the autoanalysis group [2]. For a DoA with angle $\alpha$, when its vector data $s_{\alpha} \in S$, its projection onto subspace $N$ is zero (orthogonal). Due to errors in the estimated correlation matrix, the inverse of this projection is a local maximum. Testing the DoAs of interest gives the cross-track angle estimation. The number $P$ of vectors spanning the signal subspace is the number of expected DoAs, always smaller than the number $L$ of channels. If $N$ is spanned with $Q$ vectors $n_{i}$, the estimation $D_{M U}$ is 

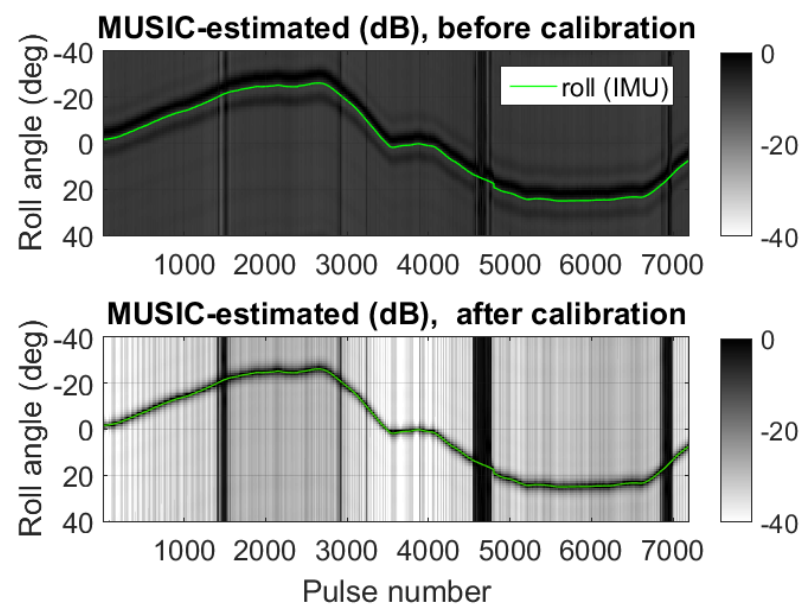

Fig. 2. Roll angle (degrees) estimated with MUSIC algorithm, from the phase at the peaks of the received signals in each RX channel, before (top) and after (bottom) calibration. The results are compared with the roll angle from the IMU (solid green).

$$
D_{M U}(\alpha)=\left(\sum_{i=1}^{Q}\left|s_{\alpha} \cdot n_{i}^{*}\right|^{2}\right)^{-1}, n_{i} \in N, i=1 \ldots Q
$$

In a uniform array with a separation lower of $\lambda / 2$, the phase difference between consecutive elements for a given DoA is constant and within the interval $(-\pi,+\pi)$. If the data vector is viewed as a signal in time domain, the normalized phase difference $f_{i}[n]$ from the $n t h$-element to the previous

$$
f_{i}[n]=\frac{1}{2 \pi}\left(\phi_{n}-\phi_{n-1}\right), \quad 2 \leq n \leq L,
$$

is analogous to the instant frequency on the normalized spectral domain of its Fourier Transform (FT). When $L \rightarrow \infty$, the spectrums of the different DoAs are not overlapped and the data vectors are orthogonal. In PASIN2, with a nonuniform array, the instant frequency is only approximately constant in port, belly and starboard sections, with different values for the same DoA, due to the wing slopes and the antenna separations for wing and belly sections. The change of phase between the elements linking the sections (wing elements closest to the fuselage and outer belly antennas, P4B5 and B8-S9) is greater than $\pi$ even for nadir DoA $\left(0^{\circ}\right)$, causing aliasing in the frequency domain of its data vector, a clear effect when calculating the FT. The element distribution causes ambiguities, since the instant frequency does not represent unequivocally the DoA, affecting the MUSIC estimation. In Fig. 3 are plotted the instant frequencies for DoAs $-30^{\circ}$ (blue) and $0^{\circ}$ (red), before (solid) and after (dashed) aliasing, the latter within the interval $[-0.5,+0.5]$. This problem is analogous to the signal identification by just its spectral power density, calculated as the FT of its timedomain correlation: for frequency-varying signals with the same density, the order of appearing of every frequency is

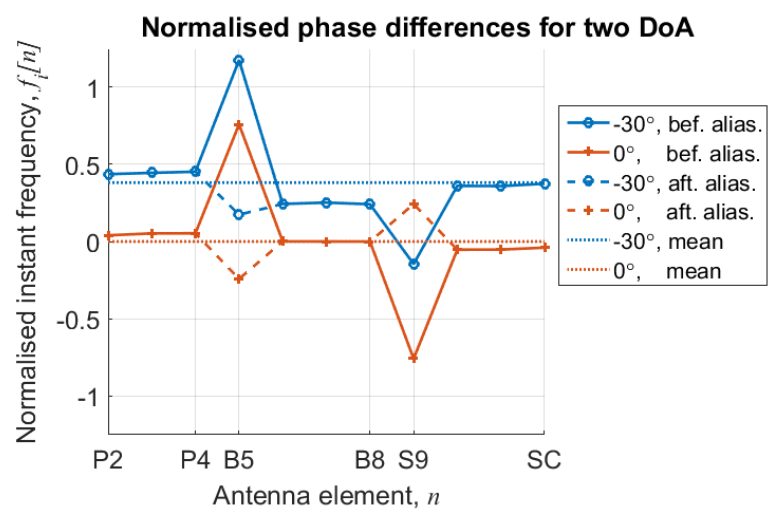

Fig. 3. Phase difference in PASIN2 RX elements, for DoAs $-30^{\circ}$ (blue) and $0^{\circ}$ (red), before aliasing (solid), after wrapping within the interval \pm 0.5 (dashed), and equivalent uniform array for the same mean values (dotted) as before aliasing.

ambiguous. To substitute the expected phase distribution of each DoA $(\alpha)$ by the equivalent for a uniform linear array with spacing $d_{E}$ along ' $y$ ' axis, improves the angular accuracy and peak amplitude. This new phase distribution is such that the instant frequency (constant, dotted line in Fig. 3) in the uniform array, $f_{i, U}$, equals the mean instant frequency (varying) in the non-linear nor uniform array, $f_{i, \alpha}[n]$

$$
f_{i, U}(\alpha)=-\frac{d_{E}}{\lambda} \sin (\alpha)=\frac{1}{L-1} \sum_{n=2}^{L} f_{i, \alpha}[n],
$$

avoiding the aliasing within the interval $\left[-41^{\circ},+41^{\circ}\right]$ in PASIN2. Using far-field approximations, $f_{i, \alpha}$ is expressed as

$$
f_{i, \alpha}[n]=-\frac{d_{n}}{\lambda} \sin \left(\beta_{n}(\alpha)\right), \quad 2 \leq n \leq L,
$$

with $\vec{p}_{n-1, n}$ the vector joining elements $n-1$ and $n$, and $\vec{q}_{\alpha}$ the unitary vector defining the DoA,

$$
\begin{aligned}
& \vec{p}_{n-1, n}=\left[x_{n}-x_{n-1}, y_{n}-y_{n-1}, z_{n}-z_{n-1}\right] \text {, }
\end{aligned}
$$

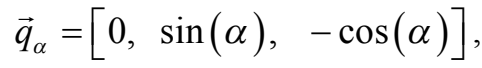

$$
\begin{aligned}
& d_{n}=\left|\vec{p}_{n-1, n}\right|, \\
& \beta_{n}(\alpha)=\cos ^{-1}\left(\frac{\vec{p}_{n-1, n}}{d_{n}} \cdot \vec{q}_{\alpha}\right)-\frac{\pi}{2}, \quad 2 \leq n \leq L .
\end{aligned}
$$

\section{RESULTS}

From (5) to (8) and the antenna coordinates found in Table I, it results $d_{E} / \lambda=0.76$. In Fig. 4 are shown the DoA estimations in decibels: in the left column, two DoAs were generated overlapping two sections of a SAR image from a data take above the sea, to build an equivalent environment with DoAs at $1.6^{\circ}$ (solid white) and $24.6^{\circ}$ (dashed white), close to a range 
2 added SAR images

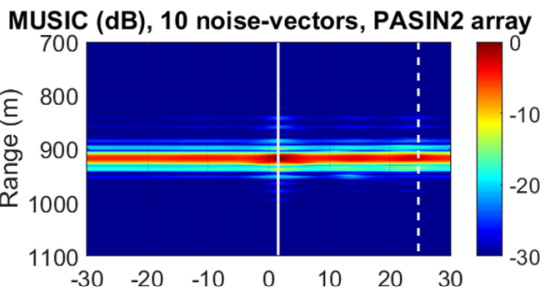

MUSIC (dB), 10 noise-vectors, uniform array

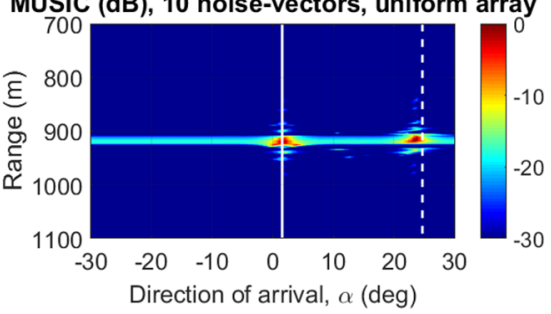

2 ideal targets

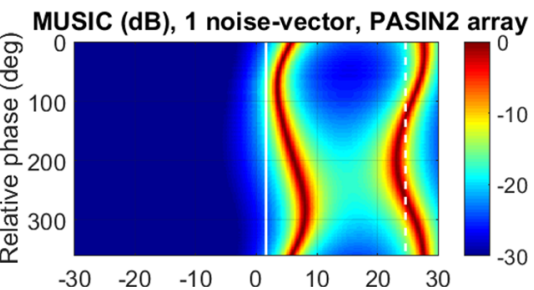

MUSIC (dB), 1 noise-vector, uniform array

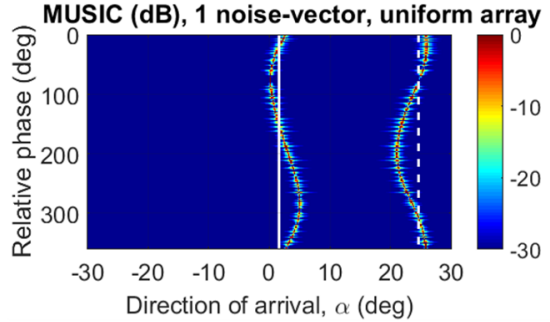

2 ideal targets

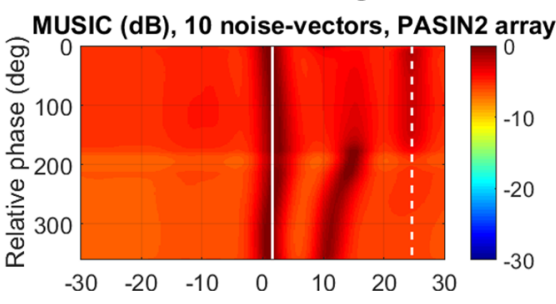

MUSIC (dB), 10 noise-vectors, uniform array

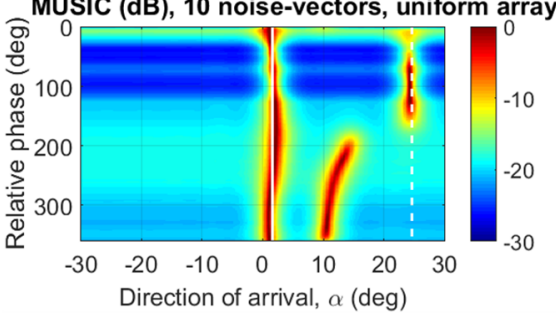

Fig. 4. DoA estimation with MUSIC (dB), for DoAs $1.6^{\circ}$ (solid) and $24.6^{\circ}$ (dashed). Left column: two overlapped regions of SAR images from a data take with a height over surface of about $900 \mathrm{~m}$, estimated with 10 noise-vectors. Middle and right column: simulated DoAs, estimated with 1 and 10-noise vectors, respectively, with the relative phase between signals in the vertical dimension. Top row: processed with data vectors from PASIN2 RX locations as references. Bottom row: processed with data vectors from the equivalent uniform array.

of 900m, corresponding to pulses 1 and 2000 in Fig. 2, with a pulse repetition frequency of $156.25 \mathrm{~Hz}$. They were processed with $Q=10$ noise vectors, the maximum for $L=12$ channels and $P=2$ expected DoAs. In the middle and right columns, the same DoAs are simulated with the ideal reference functions. Middle and right images include in the vertical axis the relative phase between DoA signals. For the estimations, top images use the ideal reference PASIN2 data vectors, while the bottom processings use the equivalent uniform array. The latter present a lower estimation floor and better angular resolution. The results vary depending on the relative phase between DoAs and the number of noise vectors applied in MUSIC: with a single noise vector (middle column) the resolution improves, but the estimations oscillate about $\pm 2.5^{\circ}$. With 10 noise vectors (right column), there is an error-step in the estimation for $\alpha=24.6^{\circ}$ when the relative phase is greater than $180^{\circ}$. As these errors are not consistent for any pair of DoAs, they cannot be calibrated. One solution could be to process a set of outputs from different correlation estimators, number of noise vectors and even channel combinations, like just contiguous channels to avoid aliasing.

\section{CONCLUSION}

The proposed procedure allows to assess the direction of arrival estimators of radar echoes from scatterers in a 3D environment, in the field of ice-sounding with airborne MIMO SAR, extending the applicability of data takes initially used for calibration and validation. With this method several point-like targets from a real data take can be included, adding arbitrary amplitudes and phases for being a flexible frame to evaluate the estimator. To overcome the problems of a non-uniform distributed array, the use of an equivalent uniform array in MUSIC estimator has been put forward, improving the bias and peak amplitude of the output.

\section{ACKNOWLEDGMENT}

We thank C.N. Robinson and R.J. Bullock for the collection and processing of raw data. This work was supported by the Natural Environment Research Council (NERC) [grant reference NE/L013444/1].

\section{REFERENCES}

[1] D. Bekaert et al., "Multichannel surface clutter suppression: East Antarctica P-band SAR ice sounding in the presence of grating lobes," Ann. Glaciol., vol. 55, no. 67, pp. 9-21, Jul. 2014.

[2] J G. Proakis, D. G. Manolakis, Digital signal processing: principles, algorithms, and applications, 3rd ed., Upper Saddle River, NJ, USA: Prentice-Hall, 1996, ch.12, sec.5.

[3] R. Schmidt, "Multiple emitter location and signal parameter estimation," IEEE Trans. Antennas Propag., vol. 34, no. 3, pp. 276-280, Mar. 1986.

[4] J. Paden, T. Akins, D. Dunson, C. Allen, and P. Gogineni, "Ice-sheet bed 3-D tomography", J. Glaciol., vol. 56, issue 195, pp. 3-11, Apr. 2010.

[5] X. Wu, K. C. Jezek, E. Rodriguez, S. Gogineni, F. Rodriguez-Morales and A. Freeman, "Ice Sheet Bed Mapping With Airborne SAR Tomography," IEEE Trans. Geosci. Remote Sens., vol. 49, no. 10, pp. 3791-3802, Oct. 2011.

[6] J. B. Yan et al., "Measurements of In-Flight Cross-Track Antenna Patterns of Radar Depth Sounder/Imager," IEEE Trans. Antennas Propag., vol. 60, no. 12, pp. 5669-5678, Dec. 2012. 